\title{
Comparison of the Effects of Ultrasound- Guided Interfascial Pulsed Radiofrequency and Ultrasound-Guided Interfascial Injection on Myofascial Pain Syndrome of the Gastrocnemius
}

\author{
So Min Park, $\mathrm{MD}^{1}$, Yun Woo Cho, $\mathrm{MD}^{2,3}$, Sang Ho Ahn, $\mathrm{MD}^{2,3}$, \\ Dong Gyu Lee, $\mathrm{MD}^{4}$, Hee Kyung Cho, $\mathrm{MD}^{5}$, Sung Yup Kim, $\mathrm{MD}^{1}$
}

\begin{abstract}
${ }^{1}$ Department of Physical Medicine and Rehabilitation, Yeungnam University College of Medicine, Daegu; ${ }^{2}$ Department of Rehabilitation Medicine \& Spine Center, Yeungnam University Medical Center, Daegu; ${ }^{3}$ Medical Devices Clinical Trial Center, Yeungnam University, Daegu; ${ }^{4}$ Department of Physical Medicine and Rehabilitation, Keimyung University School of Medicine, Daegu; ${ }^{5}$ Department of Physical Medicine and Rehabilitation, Catholic University of Daegu School of Medicine, Daegu, Korea
\end{abstract}

Objective To investigate the comparative treatment effects of ultrasound-guided pulsed radiofrequency treatment (UG-PRF) in the gastrocnemius interfascial space and ultrasound-guided interfascial injection (UG-INJ) on myofascial pain syndrome.

Methods Forty consecutive patients with myofascial pain syndrome of the gastrocnemius were enrolled and were allocated to one of the two groups. Twenty patients were treated by UG-PRF delivered to the gastrocnemius interfascial space (UG-PRF group) and the other 20 patients were treated by interfascial injection (UG-INJ group). The primary outcome measure was the numeric rating score (NRS) for pain on pressing the tender point in the gastrocnemius, and the secondary outcome measure was health-related quality of life as determined by the Short Form-36 questionnaire (SF-36). NRSs were obtained at the first visit, immediately after treatment, and at 2 and 4 weeks post-treatment, and physical component summary scores (PCS) and mental component summary scores (MCS) of the SF-36 questionnaire were measured at the first visit and at 4 weeks post-treatment.

Results Immediately after treatments, mean NRS in the UG-PRF group was significantly higher than that in the UG-INJ group $(p<0.0001)$. However, at 2 and 4 weeks post-treatment, the mean NRS was significantly lower in the UG-PRF group (both $\mathrm{p}<0.0001$ ). Similarly, at 4 weeks post-treatment, mean PCS and MCS were significantly higher in the UG-PRF group ( $\mathrm{p}<0.0001$ and $\mathrm{p}=0.002$, respectively).

Conclusion Based on these results, the authors conclude that ultrasound-guided gastrocnemius interfascial PRF provides an attractive treatment for myofascial pain syndrome of the gastrocnemius.

Keywords Pulsed radiofrequency treatment, Gastrocnemius muscle, Myofascial pain syndromes, Trigger point pain, Injections

Received January 5, 2016; Accepted March 10, 2016

Corresponding author: Sung Yup Kim

Department of Physical Medicine and Rehabilitation, Yeungnam University College of Medicine, 170 Hyeonchung-ro, Nam-gu, Daegu 42415, Korea. Tel: +82-53-620-3270, Fax: +82-53-620-3270, E-mail: yup331@hanmail.net

ORCID: So Min Park (http://orcid.org/0000-0003-0932-424X); Yun Woo Cho (http://orcid.org/0000-0002-6585-327X); Sang Ho Ahn (http://orcid. org/0000-0003-2921-7820); Dong Gyu Lee (http://orcid.org/0000-0002-4787-4448); Hee Kyung Cho (http://orcid.org/0000-0003-1950-9926); Sung Yup Kim (http://orcid.org/0000-0002-2136-6197).

(a) This is an open-access article distributed under the terms of the Creative Commons Attribution Non-Commercial License (http://creativecommons.org/ licenses/by-nc/4.0) which permits unrestricted noncommercial use, distribution, and reproduction in any medium, provided the original work is properly cited. Copyright $\odot 2016$ by Korean Academy of Rehabilitation Medicine 


\section{INTRODUCTION}

Myofascial pain occurs in about $30 \%$ of patients who attend general clinics, and an even more in the tertiary pain clinic setting [1]. This type of pain causes many functional and psychiatric problems, including anxiety and depression. In particular, myofascial pain in the gastrocnemius is a well-known cause of diminished quality of life that causes nocturnal cramps, heel pain, and restricted ankle dorsiflexion [2].

The gastrocnemius is wrapped with fascia, which is composed of undifferentiated mesenchymal tissues that wrap around or form packing material between specialized organs and tissues [3]. Muscle fascia has many functions in addition to protecting or creating osteofascial compartments for muscles and it is involved in the etiopathogenesis of numerous extra-articular pain syndromes [4]. Hence, new knowledge of the functions and anatomical arrangements of fasciae is increasingly being applied in areas like anesthesia and pain treatment [5]. During the past several decades, many types of pain relief methods, such as acupuncture, trigger point injections, stretching exercises, intramuscular electric stimulation, and physical therapies, have been developed and used to relieve myofascial pain [5-7]. Among these treatments, interfascial injection has been the mainstay of myofascial pain management for reducing acute and localized pain $[8,9]$.

Pulsed radiofrequency (PRF) treatment is a minimally neurodestructive alternative to radiofrequency heat lesions [10]. The mechanism responsible for the action of PRF remains unclear, although there is some evidence for a neuromodulatory effect [11]. PRF has been used to treat trigger points $[12,13]$. However, to the best of our knowledge, only one previous study has demonstrated the effect of PRF on the gastrocnemius for managing plantar heel pain [14].

In the present study, we compared the treatment effects of PRF and interfascial injection on myofascial pain syndrome of the gastrocnemius. Specifically, we investigated and compared the effects of ultrasound-guided pulsed radiofrequency treatment (UG-PRF) delivered to the gastrocnemius interfascial space and ultrasound-guided interfascial injection (UG-INJ).

\section{MATERIALS AND METHODS}

\section{Subjects}

This study was conducted using a prospective, randomized, and controlled clinical trial design. Patients were recruited from the rehabilitation department of Yeungnam University Medical Center from January 2015 to July 2015 using the following inclusion criteria $[15,16]$. (1) Age $\geq 18$ and $\leq 65$ years. (2) A complaint of myofascial pain in the gastrocnemius or pain in the calf area radiating to the heel and sole. Pain was not confined to one dermatome or myotome, and physical examinations revealed taut bands and one or more identifiable trigger points in the gastrocnemius. (3) Symptoms that had persisted for at least 3 months. (4) Normal neurological examination findings for deep tendon reflexes, manual muscle tests, and sensory examinations. (5) The absence of other diagnosed conditions, such as lumbar radiculopathy or nerve entrapment syndromes. Based on the patient's history and physical examination, additional workup such as lumbar spine magnetic resonance imaging and electromyography was performed if distinction between myofascial pain in the gastrocnemius and other diagnosed conditions was not clear. And (6) the presence of pain on pressing the tender point in the gastrocnemius, rated at $\geq 4$ points on the numeric rating scale (NRS). The exclusion criteria applied were as follows: (1) pregnancy; (2) the presence of coagulopathy, or the use of anticoagulants; (3) an inability to understand the instructions or complete the questionnaire; (4) gastrocnemius muscle pain caused by inflammatory, malignant, or autoimmune disease; (5) the presence of a chronic medical condition that might preclude participation in the study, such as malignancy, systemic inflammatory disorder, a neurological abnormality, sciatica, and/or chronic pain; (6) a history of surgery on the gastrocnemius muscle; and (7) known hypersensitivity to metals. Forty patients (21 males, 19 females; mean age, 50.1 \pm 9.7 years; range, 34-65 years) were recruited. Informed consent was obtained from all participants, and the study protocol was approved beforehand by the Institutional Review Board of Yeungnam University Medical Center.

\section{Randomization}

The 40 study subjects with myofascial pain syndrome of the gastrocnemius muscle were assigned to two equally 
sized groups, i.e., the UG-PRF group or the UG-INJ group, by a nurse using a random table. Subjects in both groups were requested not to use any oral medication during the 4-week study period.

\section{Intervention}

To increase the accuracy of PRF and interfascial injection, ultrasound imaging was used to avoid unintentional damage to these important structures, with no irradiation delivered to the patients.

Briefly, PRF was performed with the patient in the prone position. The tender point was established physically (a hypersensitive bundle or nodule of muscle fiber of harder than normal consistency on palpitation) $[17,18]$. The skin over the tender point was marked and sterilized by Hexial $2 \%$ solution (ethanol+chlorhexidine gluconate $2 \%$ ), and a sterile surgical towel was placed on the patient. A linear array transducer probe (LOGIQ P6; GE
Healthcare, Waukesha, WI, USA) was used to scan the marked area in the sagittal and axial planes. Initially, the probe was used to identify the gastrocnemius and its fascia. After taking all preparatory steps mentioned above, a local anesthetic ( $1 \%$ lidocaine) was administered by skin infiltration, and then, under ultrasound guidance, a 22-gauge $10 \mathrm{~cm}$ needle with a 5-mm tip Cosman radiofrequency cannula (CC10522; Cosman Medical, Burlington, MA, USA) was inserted into the gastrocnemius interfascial space. Before starting PRF, $5 \mathrm{~mL}$ of normal saline was infused through the needle to separate the gastrocnemius interfascial space for identification purpose and to ensure correct needle positioning (Fig. 1). After the PRF needle was connected to a PRF electrode, the interfascial area was treated by a Cosman G4 radiofrequency generator (Cosman Medical). The following modes were used: (1) maximal temperature $42^{\circ} \mathrm{C}$, (2) duration 6 minutes, (3) voltage $55 \mathrm{~V}$, (4) pulse rate $5 \mathrm{~Hz}$, and (5) pulse width 5
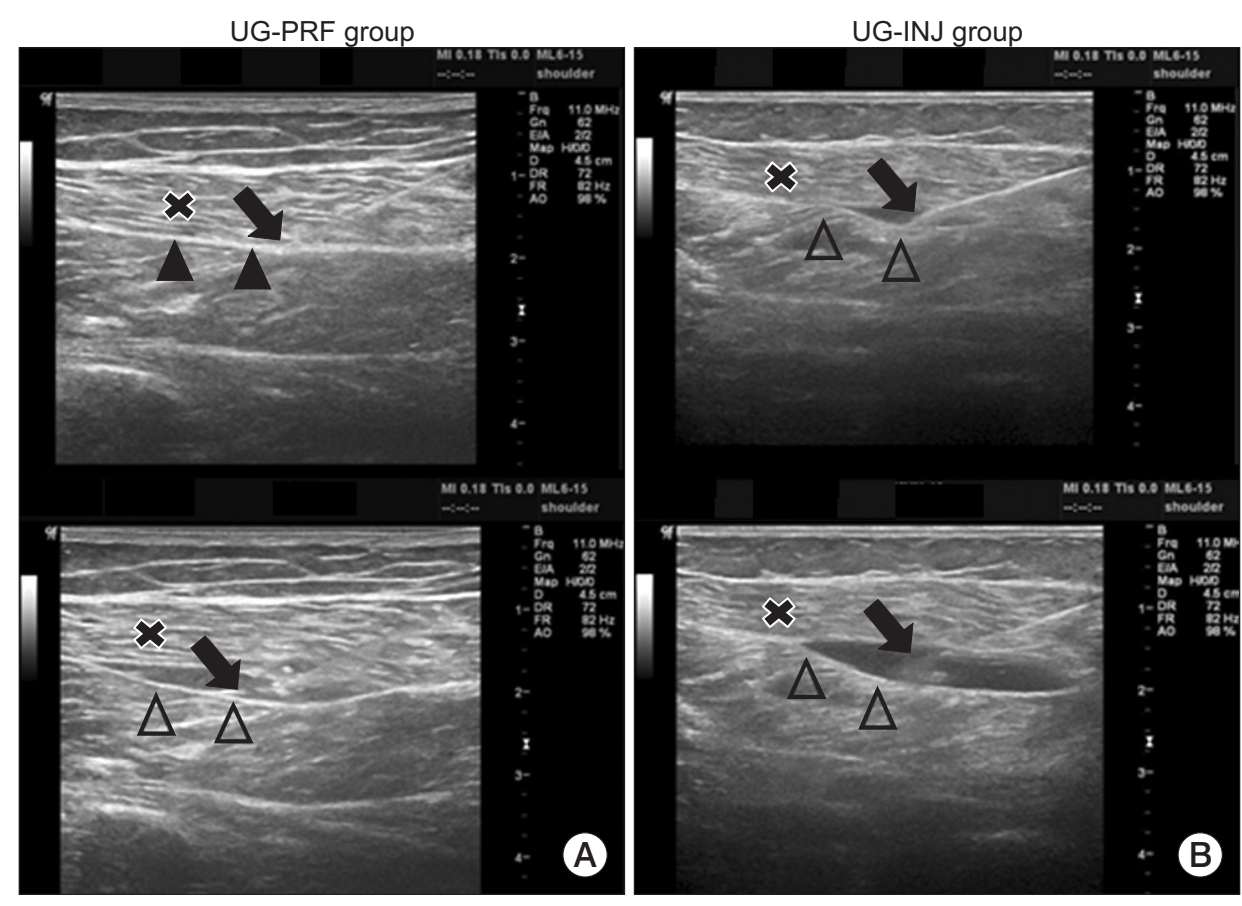

Fig. 1. Ultrasound images showing the needle point in the interfascial space of UG-PRF group and UG-INJ group. (A) UG-PRF group: the probe identified the gastrocnemius (x) and its fascia (arrow head). A 22 -gauge $10 \mathrm{~cm}$ needle with a 5-mm tip was inserted into the gastrocnemius interfascial space (arrow). Before starting PRF, $5 \mathrm{~mL}$ normal saline was infused through the needle and it separated the gastrocnemius interfascial space to better identify that space and better position the needle into that space (hollow arrow head). (B) The UG-INJ group: the probe identified the gastrocnemius (x) and its fascia (arrow head). A 22-gauge $10 \mathrm{~cm}$ needle with a 5-mm tip was inserted into the gastrocnemius interfascial space (arrow). Under ultrasound guidance, $10 \mathrm{~mL}$ of $0.2 \%$ lidocaine was injected into the gastrocnemius interfascial space (hollow arrow head). UG-PRF, ultrasound-guided pulsed radiofrequency treatment; UG-INJ, ultrasound-guided interfascial injection. 
ms. When a patient had more than one tender point, the procedure was repeated at each tender point.

The preparatory steps used in the UG-INJ group were identical to those described above for the UG-PRF group, and under ultrasound guidance, $10 \mathrm{~mL}$ of $0.2 \%$ lidocaine was injected into the gastrocnemius interfascial space around the tender point using an identical PRF needle [5] (Fig. 1). When a patient had more than one tender point, the procedure was repeated at each tender point, but only up to $20 \mathrm{~mL}$ of injection was administered regardless of the number of tender points.

Patients did not receive any medication after treatment and were discharged on the same day. PRF treatment and interfascial injection were administered by the same physician, but assessments and follow-up were conducted by other investigators who were not aware of group assignments.

\section{Outcomes measures}

As a primary outcome measure, we measured pain intensity of tenderness on the gastrocnemius muscle using an 11-point NRS (0, no pain; 10, the worst pain imaginable) [19]. If there was more than one tender point, we used the NRS value of the most severe tender point. The secondary outcome measures were the Health Survey physical component summary (PCS) and mental component summary (MCS) subscores of Short Form 36 (SF-36; each measured on a 0- to 100-point scale, where higher scores indicate better health) [20]. Blinded assessments were made by a medical specialist. The NRS was obtained at the first visit to the clinic, immediately after treatment, and at 2 and 4 weeks post-treatment, and PCS and MCS were measured at the first visit and at 4 weeks post-treatment. Possible adverse events were assessed by the medical specialist on days $1,7,14,21$, and 28 after treatment.

\section{Statistical analysis}

The analysis was performed using SPSS ver. 17.0 software (SPSS Inc., Chicago, IL, USA). The Mann-Whitney test and the chi-square test were used to determine the significance of differences between the UG-PRF and UGINJ groups, and the Wilcoxon test and the Friedman test were used to analyze individual score changes. Statistical significance was accepted for $\mathrm{p}$-values of $<0.05$.

\section{RESULTS}

Forty patients aged 34 to 65 years were enrolled, and all patients successfully completed the follow-up. Age, sex, the number of tender points, pain severity (NRS) and the PCS and MCS of SF-36 were not significantly different between the UG-PRF and UG-INJ groups. Patient characteristics are provided in Table 1.

Mean NRSs in both groups were significantly lower immediately after treatment than pre-treatment (mean NRS $5.0 \pm 0.7$ and $2.0 \pm 0.6$ in the UG-PRF and UG-INJ groups immediately after treatment; $\mathrm{p}=0.008$ and $\mathrm{p}<0.0001$ in the UG-PRF and UG-INJ groups). In the UG-PRF group, mean NRS at 2 weeks post-treatment was significantly lower than that immediately after treatment (mean NRS $2.6 \pm 0.9$ at 2 weeks post-treatment; $\mathrm{p}<0.0001$ ). However, mean NRSs at 2 and 4 weeks post-treatment in the UGPRF group were not significantly different (mean NRS $2.4 \pm 1.0$ at 4 weeks post-treatment; $\mathrm{p}=0.302$ ), and mean PCS and MCS at 4 weeks post-treatment were significantly higher than those pre-treatment (mean PCS and MCS $41.7 \pm 7.3$ and $44.0 \pm 5.9$ at 4 weeks post-treatment; $\mathrm{p}<0.0001)$. On the other hand, in the UG-INJ group, mean NRS at 2 weeks post-treatment was significantly higher than that immediately after treatment (mean NRS $4.3 \pm 0.9$ at 2 weeks post-treatment; $\mathrm{p}<0.0001)$, but at 4 weeks

Table 1. Demographic characteristics of the UG-PRF and UG-INJ groups

\begin{tabular}{lccc}
\hline & $\begin{array}{c}\text { UG-PRF } \\
\text { group } \\
(\mathbf{n = 2 0})\end{array}$ & $\begin{array}{c}\text { UG-INJ } \\
\text { group } \\
(\mathbf{n = 2 0 )}\end{array}$ & $\begin{array}{c}\text { p- } \\
\text { value }\end{array}$ \\
\hline Age (yr) & $48.9 \pm 8.5$ & $51.3 \pm 10.0$ & 0.4 \\
Sex (male:female) & $11: 9$ & $10: 10$ & \\
\hline Number of tender points & $2.4 \pm 0.5$ & $2.3 \pm 0.4$ & 0.8 \\
NRS (pre-treatment) & $5.5 \pm 0.9$ & $5.2 \pm 1.0$ & 0.4 \\
SF-36 & & & \\
PCS & $31.0 \pm 4.5$ & $32.0 \pm 3.3$ & 0.1 \\
\hline MCS & $34.8 \pm 3.7$ & $36.8 \pm 3.5$ & 0.06 \\
\hline
\end{tabular}

Values are presented as mean \pm standard deviation or number.

UG-PRF, ultrasound-guided pulsed radiofrequency treatment; UG-INJ, ultrasound-guided interfascial injection; NRS, numeric rating scale; SF-36, Short Form-36; PCS, physical component summary score of SF-36; MCS, mental component summary score of SF-36. 
post-treatment, mean NRS was not significantly higher than that at 2 weeks post-treatment (mean NRS $4.0 \pm 0.8$ at 4 weeks post-treatment; $\mathrm{p}=0.058)$. Mean PCS and MCS in the UG-INJ group were significantly higher at 4 weeks post-treatment than pre-treatment (mean PCS and MCS $32.9 \pm 2.8$ and $37.8 \pm 3.6$ at 4 weeks post-treatment; $\mathrm{p}<0.0001)$.

Immediately after treatment, mean NRS was significantly higher in the UG-PRF group than in the UG-INJ group (mean NRS 5.0 \pm 0.7 and $2.0 \pm 0.6$ in the UG-PRF and UG-INJ groups; $\mathrm{p}<0.0001$ ), but at 2 weeks post-treatment, mean NRS was significantly lower in the UG-PRF group (mean NRS $2.6 \pm 0.9$ and $4.3 \pm 0.9$ in the UG-PRF and UGINJ groups; $\mathrm{p}<0.0001$ ). At 4 weeks post-treatment, mean NRS was significantly lower in the UG-PRF group than in the UG-INJ group (mean NRS $2.4 \pm 1.0$ and $4.0 \pm 0.8$ in the
UG-PRF and UG-INJ groups, $\mathrm{p}<0.0001$ ); similarly, mean PCS (mean PCS $41.7 \pm 7.3$ and $32.9 \pm 2.8$ in the UG-PRF and UG-INJ groups, $\mathrm{p}<0.0001$ ) and mean MCS (mean MCS $44.0 \pm 5.9$ and $37.8 \pm 3.6$ in the UG-PRF and UG-INJ groups; $\mathrm{p}=0.002$ ) were significantly higher in the UG-PRF group. Adverse events were observed in the UG-INJ group immediately after treatment; 2 patients complained of dizziness and nausea. Results are summarized in Table 2. Fig. 2 presents the change in mean NRSs between the UG-PRF and UG-INJ groups over time.

\section{DISCUSSION}

The results of this randomized trial show that UG gastrocnemius interfascial PRF has longer term effects on myofascial pain syndrome of the gastrocnemius, as de-

Table 2. Outcomes of the UG-PRF and UG-INJ groups

\begin{tabular}{|c|c|c|c|}
\hline & UG-PRF group & UG-INJ group & p-value \\
\hline \multicolumn{4}{|l|}{ NRS } \\
\hline Pre-treatment & $5.5 \pm 0.9$ & $5.2 \pm 1.0$ & 0.40 \\
\hline Immediately after treatment & $5.0 \pm 0.7$ & $2.0 \pm 0.6$ & $<0.001^{* * *}$ \\
\hline 2 weeks & $2.6 \pm 0.9$ & $4.3 \pm 0.9$ & $<0.001^{* * *}$ \\
\hline 4 weeks & $2.4 \pm 1.0$ & $4.0 \pm 0.8$ & $<0.001^{* * *}$ \\
\hline \multicolumn{4}{|l|}{ p-value } \\
\hline Pre-treatment vs. immediately after treatment & $0.008^{* *}$ & $<0.001^{* * *}$ & \\
\hline Immediately after treatment vs. 2 weeks & $<0.001^{* * *}$ & $<0.001^{* * *}$ & \\
\hline 2 weeks vs. 4 weeks & 0.3 & 0.05 & \\
\hline \multicolumn{4}{|l|}{ SF-36 } \\
\hline \multicolumn{4}{|l|}{ PCS } \\
\hline Pre-treatment & $31.0 \pm 4.5$ & $32.0 \pm 3.3$ & 0.10 \\
\hline 4 weeks & $41.7 \pm 7.3$ & $32.9 \pm 2.8$ & $<0.001^{* * *}$ \\
\hline p-value & $<0.001^{* * *}$ & $<0.001^{* * *}$ & \\
\hline \multicolumn{4}{|l|}{ MCS } \\
\hline Pre-treatment & $34.8 \pm 3.7$ & $36.8 \pm 3.5$ & 0.06 \\
\hline 4 weeks & $44.0 \pm 5.9$ & $37.8 \pm 3.6$ & $0.002^{* *}$ \\
\hline p-value & $<0.001^{* * *}$ & $<0.001^{* * *}$ & \\
\hline \multicolumn{4}{|l|}{ Adverse events } \\
\hline Dizziness and nausea & 0 & 2 & \\
\hline
\end{tabular}

Values are presented as mean \pm standard deviation or number.

UG-PRF, ultrasound-guided pulsed radiofrequency treatment; UG-INJ, ultrasound-guided interfascial injection; NRS, numeric rating scale; SF-36, Short Form-36; PCS, physical component summary score of the SF-36; MCS, mental component summary score of the SF-36.

Statistically significant at the $\mathrm{p}=0.05$ level.

${ }^{* *} \mathrm{p}<0.01,{ }^{* * *} \mathrm{p}<0.001$. 


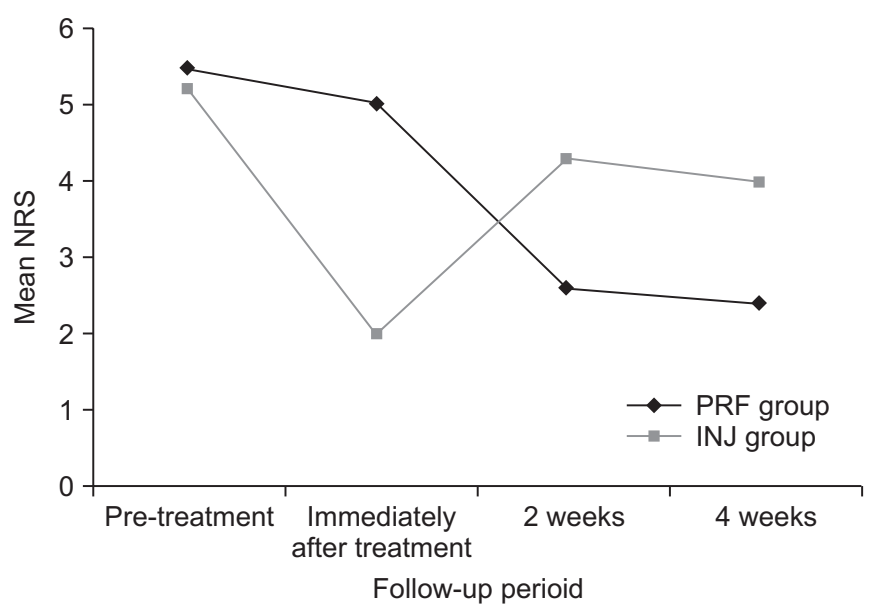

Fig. 2. Change in mean numeric rating scales between the UG-PRF and UG-INJ groups over time. NRS, numeric rating score; UG-PRF, ultrasound-guided pulsed radiofrequency treatment; UG-INJ, ultrasound-guided interfascial injection.

termined by NRSs, PCSs, and MCSs, than UG gastrocnemius interfascial injection. Although UG gastrocnemius interfascial PRF was found to be significantly less effective immediately after treatment than UG gastrocnemius interfascial injection, from 2 weeks after PRF treatment, it was significantly more effective than interfascial injection. In addition, two mild adverse events were observed in the UG-INJ group. We thought that gastrocnemius interfascial injection had a better effect immediately after treatment due to local anesthetics. In contrast, we made the following inferences regarding why gastrocnemius interfascial PRF had a slower and longer effect.

Fasciae have many functions, for example, they create distinctive compartments for muscles, provide circulatory support and protection [3]. Recently, several authors have reported that interfascial block provides myofascial pain relief $[5,8,21,22]$. Domingo et al. [5] studied ultrasound-guided interfascial block of the trapezius muscle, and they found in a histological study that the interfascial space possesses many piercing nerve branches related to myofascial pain, which explains why interfascial local anesthesia provides some relief from myofascial pain.

$\mathrm{PRF}$ is a minimally neurodestructive alternative that has been used to treat many types of chronic pain [23]. However, its healing effect is poorly understood. Nonetheless, some studies have been undertaken to investigate the therapeutic mechanism underlying the effects of PRF
[24-26]. Erdine et al. [24] used electron microscopy and studied the structural effects of PRF on sensory nociceptive axons, and they noted that exposure to PRF caused ultrastructural damage to axons, that affected smaller principal sensory nociceptors, C-fibers, and A-delta fibers more than larger non-pain related sensory fibers, such as A-beta fibers. Moffett et al. [25] conducted a study on the therapeutic mechanism of PRF in the genetic aspect. They said that the PRF energy fields could increase the level of endogenous opioid precursor mRNA and the corresponding opioid peptide. Similarly, in an animal study of PRF treated peripheral nerve injury, Vallejo et al. [26] found gene modulation in multiple neuronal tissues adjacent to sites of injury. In addition, it was observed that PRF regulated pro-inflammatory gene expression at sites of injury and in dorsal root ganglions and spinal cords. Based on the findings of these previous studies with respect to the therapeutic mechanism of PRF, we concluded that PRF may have affected the nerve branches passing through the interfascial space and caused selective blocking of nerve fibers, especially C-fibers and Adelta fibers. We thought that this was the reason why PRF had a slow effect and a long-lasting therapeutic effect on myofascial pain syndrome.

Several studies used PRF to treat myofascial pain syndrome. Bevacqua and Fattouh [12] studied the effect of PRF on painful trigger points in ten patients who suffered from myofascial pain syndrome. Tamimi et al. [13] introduced PRF treatment of myofascial trigger points and scar neuromas in 9 patients. These studies revealed pain relief by using PRF treatment for trigger points in the muscle. However, these studies had some limitations; they had a very small sample size and there was no control group. In a recent study, Ye et al. [14] studied PRF treatment in the gastrocnemius for managing plantar heel pain compared with sham treatment. They applied PRF treatment to the trigger point of the muscle and found that inactivation of trigger points relieved gastrocnemius contracture and plantar heel pain. It is meaningful that this is the first study in which PRF treatment was applied to the muscle. Compared to previous studies, the present study is significant as the interfascial space was targeted by PRF treatment and the effect of interfascial PRF was compared with that of interfascial injection.

Some limitations of the present study warrant consideration. First, it was designed as a single center study and 
it had a small sample size. Second, the follow-up period was short of only 4 weeks, and thus, the long-term effect of PRF could not be determined. Third, the study lacked a control group to circumvent questions regarding therapeutic effects versus spontaneous symptom resolution and it was not a double blind study due to the difference between two treatments. In the next study, it is necessary to compare the PRF treatment group and the sham treatment group. Fourth, the study subjects were enrolled at a university hospital and were more likely to have severe symptoms. Fifth, in this study, the explanation for the therapeutic effect and impact range of interfascial PRF was insufficient. In order to achieve a greater persuasive power about our conclusion, further research on the nerve in the interfascial space and PRF effects is needed.

Nevertheless, this is the first study on the use of interfascial PRF delivered to the gastrocnemius to treat myofascial pain syndrome of the gastrocnemius. Notably, ultrasound-guided interfascial PRF delivered to the gastrocnemius was found to have a longer effect and to be safer than ultrasound-guided interfascial injection. Based on these results, it would appear that interfascial PRF delivered to the gastrocnemius provides an alternative treatment for myofascial pain syndrome of the gastrocnemius.

\section{CONFLICT OF INTEREST}

No potential conflict of interest relevant to this article was reported.

\section{ACKNOWLEDGMENTS}

This study was supported by a grant of the Korea Healthcare Technology R\&D Project by Ministry for Health, Welfare \& Family Affairs, Korea (No. A084177).

\section{REFERENCES}

1. Skootsky SA, Jaeger B, Oye RK. Prevalence of myofascial pain in general internal medicine practice. West $J$ Med 1989;151:157-60.

2. Simons DG, Mense S. Understanding and measurement of muscle tone as related to clinical muscle pain. Pain 1998;75:1-17.

3. Benjamin M. The fascia of the limbs and back: a re- view. J Anat 2009;214:1-18.

4. Stecco A, Macchi V, Masiero S, Porzionato A, Tiengo C, Stecco C, et al. Pectoral and femoral fasciae: common aspects and regional specializations. Surg Radiol Anat 2009;31:35-42.

5. Domingo T, Blasi J, Casals M, Mayoral V, Ortiz-Sagrista JC, Miguel-Perez M. Is interfascial block with ultrasound-guided puncture useful in treatment of myofascial pain of the trapezius muscle? Clin J Pain 2011;27:297-303.

6. Hong CZ. Lidocaine injection versus dry needling to myofascial trigger point: the importance of the local twitch response. Am J Phys Med Rehabil 1994;73:25663.

7. Chu J, Yuen KF, Wang BH, Chan RC, Schwartz I, Neuhauser D. Electrical twitch-obtaining intramuscular stimulation in lower back pain: a pilot study. Am J Phys Med Rehabil 2004;83:104-11.

8. McDonnell JG, O'Donnell B, Curley G, Heffernan A, Power C, Laffey JG. The analgesic efficacy of transversus abdominis plane block after abdominal surgery: a prospective randomized controlled trial. Anesth Analg 2007;104:193-7.

9. Hebbard P. Subcostal transversus abdominis plane block under ultrasound guidance. Anesth Analg 2008; 106:674-5.

10. Van Zundert J, de Louw AJ, Joosten EA, Kessels AG, Honig W, Dederen PJ, et al. Pulsed and continuous radiofrequency current adjacent to the cervical dorsal root ganglion of the rat induces late cellular activity in the dorsal horn. Anesthesiology 2005;102:125-31.

11. Abejon D, Reig E. Is pulsed radiofrequency a neuromodulation technique? Neuromodulation 2003;6:1-3.

12. Bevacqua B, Fattouh M. Pulsed radiofrequency for treatment of painful trigger points. Pain Pract 2008; 8:149-50.

13. Tamimi MA, McCeney MH, Krutsch J. A case series of pulsed radiofrequency treatment of myofascial trigger points and scar neuromas. Pain Med 2009;10:1140-3.

14. Ye L, Mei Q, Li M, Gu M, Ai Z, Tang K, et al. A comparative efficacy evaluation of ultrasound-guided pulsed radiofrequency treatment in the gastrocnemius in managing plantar heel pain: a randomized and controlled trial. Pain Med 2015;16:782-90.

15. Simons DG. New views of myofascial trigger points: etiology and diagnosis. Arch Phys Med Rehabil 2008; 
89:157-9.

16. Simons DG, Travell JG, Simons LS. Travell \& Simons' myofascial pain and dysfunction: the trigger point manual. 2nd ed. Baltimore: Williams \& Wilkins; 1999.

17. Jaeger B. Myofascial trigger point pain. Alpha Omegan 2013;106:14-22.

18. Shankar H, Reddy S. Two- and three-dimensional ultrasound imaging to facilitate detection and targeting of taut bands in myofascial pain syndrome. Pain Med 2012;13:971-5.

19. Farrar JT, Young JP Jr, LaMoreaux L, Werth JL, Poole RM. Clinical importance of changes in chronic pain intensity measured on an 11-point numerical pain rating scale. Pain 2001;94:149-58.

20. Ware JE Jr. SF-36 health survey update. Spine (Phila Pa 1976) 2000;25:3130-9.

21. French JL, McCullough J, Bachra P, Bedforth NM. Transversus abdominis plane block for analgesia after caesarean section in a patient with an intracranial le- sion. Int J Obstet Anesth 2009;18:52-4.

22. Tran TM, Ivanusic JJ, Hebbard P, Barrington MJ. Determination of spread of injectate after ultrasoundguided transversus abdominis plane block: a cadaveric study. Br J Anaesth 2009;102:123-7.

23. Snidvongs S, Mehta V. Pulsed radio frequency: a nonneurodestructive therapy in pain management. Curr Opin Support Palliat Care 2010;4:107-10.

24. Erdine S, Bilir A, Cosman ER, Cosman ER. Ultrastructural changes in axons following exposure to pulsed radiofrequency fields. Pain Pract 2009;9:407-17.

25. Moffett J, Fray LM, Kubat NJ. Activation of endogenous opioid gene expression in human keratinocytes and fibroblasts by pulsed radiofrequency energy fields. J Pain Res 2012;5:347-57.

26. Vallejo R, Tilley DM, Williams J, Labak S, Aliaga L, Benyamin RM. Pulsed radiofrequency modulates pain regulatory gene expression along the nociceptive pathway. Pain Physician 2013;16:E601-13. 